\title{
Phonon Transport Across Coherent and Incoherent Interfaces
}

\author{
WEIXUAN LI (i), ${ }^{1,4}$ XIANG CHEN,$^{2}$ and SHENGFENG YANG ${ }^{3}$ \\ 1.-Department of Mechanical and Aerospace Engineering, University of Florida, Gainesville, \\ FL 32611, USA. 2.-Institute for Micromanufacturing, Mechanical Engineering, Louisiana Tech \\ University, Ruston, LA 71272, USA. 3.-Department of Mechanical and Energy Engineering, \\ Indiana University-Purdue University Indianapolis, Indianapolis, IN 46202, USA. 4.-e-mail: av- \\ erylwx@ufl.edu
}

\begin{abstract}
Phonon-mediated heat transport in two-dimensional superlattices with coherent and incoherent interfaces is simulated by using the concurrent atomistic-continuum method. The energy transmission across superlattices with incoherent interfaces is found to be an order of magnitude lower than that with coherent interfaces. The simulation results provide a direct visualization of the transient processes of phonon propagation and scatterings, which facilitates an improved mechanistic understanding of phonon transport across multiple interfaces. This work finds that heat conduction in superlattices with coherent interfaces is dominated by coherent phonons, while the existence of defects that comprise the incoherent interfaces destroys the wave interference and changes the phonon transport nature from coherent to diffusive. In addition, phonon scattering by interface defects becomes stronger with decreasing phonon wavelength, and the phonon coherence is destroyed for phonons with $5 \mathrm{~nm}$ wavelength.
\end{abstract}

\section{INTRODUCTION}

The behavior of thermal transport across interfaces, often characterized as interfacial thermal conductance, plays an important role in determining the overall thermal conductivity of a material system, especially when its size is comparable to the phonon mean free path. ${ }^{1}$ The thermal conductance of material interfaces has been extensively studied by experiments ${ }^{2-7}$ and is found to span over three orders of magnitude. ${ }^{1}$ On interfaces between two disparate materials with mismatched lattice constants, the formation of interface defects such as misfit dislocations is energetically favorable when the layer thickness exceeds a critical value that ranges from a few nanometers to several tens of nanometers. Interface defects are studied by experiments and shown to cause a significant reduction in the thermal conductivity of superlattices. Experimental work by Lee et al. ${ }^{8}$ shows that thermal conductivity of $\mathrm{Si} / \mathrm{Ge}$ superlattices drastically drops at period thickness around $130 \AA{ }^{8}$ which cannot be explained by theoretical models that assume the interfaces to be perfect. Instead, they suggest that defects on interfaces are responsible for such a thermal conductivity reduction. Zhu et al. ${ }^{9}$ studied phonon transport in graphene/b-BN superlattices using non-equilibrium molecular dynamics. This study shows that the thermal conductivity can be 4.25 times lower when the superlattice contains interface defects. In contrast, with multiple interfaces, the effect of interface defects on phonon transport across a single interface is found to be much less significant. As measured by Costescu et al., ${ }^{6}$ for the thermal conductance of $\mathrm{TiN} / \mathrm{MgO}$ (001) with a coherent interface, $\mathrm{TiN} / \mathrm{MgO}$ (111) with a low density of defects and $\mathrm{TiN} / \mathrm{Al}_{2} \mathrm{O}_{3}$ with a high density of defects, no significant difference was found. As shown by Hanisch-Blicharski et al., ${ }^{10}$ thermal conductance of the $\mathrm{Bi} / \mathrm{Si}$ interface with and without interface defects differs $<7 \%$; the role of interface defects appears trivial. The work by Hopkins et al. ${ }^{11}$ suggests a minor effect of interface defect density on thermal conductance across GaSb/ GaAs interfaces. It is shown that only with a second order of magnitude change in the defect density does the thermal conductance change by a factor of two. ${ }^{1}$ Existing works show that the roles of interface defects are qualitatively distinct for phonon transport across single and multiple interfaces, and the difference cannot be explained by a superposition of individual interfaces, which implies a change of the 
phonon transport nature. In the simulation work by Zhu et al. ${ }^{9}$ on $\mathrm{g} / \mathrm{b}-\mathrm{BN}$ superlattices with coherent interfaces, a minimum thermal conductivity exists at a critical period thickness. Similar results have also been found in other existing works on thermal transport in superlattices, ${ }^{12-14}$ implying a crossover between coherent and incoherent phonon scatterings. For $\mathrm{g} / \mathrm{b}-\mathrm{BN}$ superlattices containing interface defects, the minimum value disappears and the thermal conductivity increases with the period thickness monotonically. ${ }^{9}$ In most of existing works, thermal transport across interfaces is studied at steady state; the phonon transport nature is understood merely through the interpretation of measured thermal conductivity or thermal conductance. To gain a detailed mechanistic understanding of phonon transport across coherent and incoherent interfaces, a study of the transient processes of phonon propagation and scatterings is necessary.

Applications of experimental techniques such as time domain thermo-reflectance (TDTR) utilizing ultrafast laser pulses enable precise measurements of interfacial thermal conductance and provide insights into the phonon transport nature. ${ }^{15}$ It is however difficult for experiments to directly capture the transient processes of phonon propagation or scattering. Computational approaches to the study of phonon transport include atomic green function (AGF), ${ }^{16}$ the Boltzmann transport equation (BTE) ${ }^{17}$ lattice dynamics (LD) ${ }^{18}$ and molecular dynamics (MD). Different from methods such as AGF, BTE and $\mathrm{LD}$, which require prior understandings of phonon properties, i.e., coherent or incoherent, in MD simulations, phonon propagation and scattering phenomena naturally emerge as consequences of Newton's law of mechanics and interatomic potentials. A limitation of $\mathrm{MD}$ is that the maximum phonon wavelength and mean free path are cut off by the simulation cell, which is typically on the nanoscale; this size limit cannot be overcome by applying periodic boundary conditions. ${ }^{19}$ In MD simulations, there is a lack of information on phonons with long wavelength or long mean free path, whose contributions can be nontrivial. ${ }^{20,21}$ To include these phonons, and to have a superlattice model with at least ten interfaces to allow the phonon wave interference, ${ }^{22}$ a micron-scale computer model is required.

In this work, we use the concurrent atomisticcontinuum (CAC) method to study the role of interface defects on phonon transport across multiple interfaces in superlattices. Based on theoretical foundations by Chen, ${ }^{23-26}$ CAC is pioneered by Xiong ${ }^{27}$ and further developed by Deng, ${ }^{28}$ Yang ${ }^{29}$ and $\mathrm{Xu} ; 30,31$ the methodology has been demonstrated effective in simulating material systems from the nano- to mesoscales. ${ }^{31-47}$ Similar to MD, CAC does not require assumptions or empirical laws regarding phonon properties; it is therefore predictive in simulating phonon transport at the micron scale.
The objectives of this work are two-fold: first, to simulate phonon transport in superlattices with coherent and incoherent interfaces, seeking a direct visualization of the process of phonon propagation and scatterings; second, to quantify the effect of interface defects on phonon transport across superlattices by calculating energy transmission. The rest of the article is organized as follows. After an introduction of CAC methodology, computer models of superlattices with coherent and incoherent interfaces, as well as the simulation setup, are presented; simulation results of phonon transport in superlattices are then presented and discussed; the article concludes with a brief summary.

\section{CAC METHODOLOGY}

CAC is a coarse-grained atomistic model that is able to reduce degrees of freedom (DOFs) of the atomistic system. Fundamental to CAC is an extension of Irving and Kirkwood's statistical mechanical theory of transport processes ${ }^{48,49}$ to the two-level material description, which is consistent with solidstate physics. ${ }^{50}$ It leads to a concurrent atomisticcontinuum representation of the balance laws of mass, linear momentum and energy, ${ }^{51}$ which are exact consequences of Newton's second law, i.e.,

$$
\begin{aligned}
& \frac{\mathrm{d} \rho^{\alpha}}{\mathrm{d} t}+\rho^{\alpha}\left(\nabla_{x} \cdot \mathbf{v}+\nabla_{y^{\alpha}} \cdot \Delta \mathbf{v}^{\alpha}\right)=0 \\
& \rho^{\alpha} \frac{\mathrm{d}}{\mathrm{d} t}\left(\mathbf{v}+\Delta \mathbf{v}^{\alpha}\right)=\nabla_{x} \cdot \mathbf{t}^{\alpha}+\nabla_{y^{\alpha}} \cdot \tau^{\alpha}+\mathbf{f}_{\mathrm{ext}}^{\alpha} \\
& \frac{\mathrm{d}}{\mathrm{d} t}\left(\rho^{\alpha} e^{\alpha}\right)=\nabla_{x} \cdot \mathbf{q}^{\alpha}+\nabla_{y^{\alpha}} \cdot \mathbf{j}^{\alpha}+\mathbf{t}^{\alpha}: \nabla_{x}\left(\mathbf{v}+\Delta \mathbf{v}^{\alpha}\right) \\
& \quad+\tau^{\alpha}: \nabla_{y^{\alpha}}\left(\mathbf{v}+\Delta \mathbf{v}^{\alpha}\right)
\end{aligned}
$$

where $\mathbf{x}$ is the physical space coordinate of the continuously distributed lattice; $\mathbf{y}^{\alpha}$ is the relative position of atom $\alpha$ to the mass center of the lattice located at $\mathbf{x} ; \rho^{\alpha}, \rho^{\alpha}\left(\mathbf{v}+\Delta \mathbf{v}^{\alpha}\right)$ and $\rho^{\alpha} e^{\alpha}$ are the local densities of mass, linear momentum and total energy, respectively; $\left(\mathbf{v}+\Delta \mathbf{v}^{\alpha}\right)$ is the atomic-level velocity and $\mathbf{v}$ is the velocity field; $\mathbf{f}_{\mathrm{ext}}^{\alpha}$ is the external force field; $\mathbf{t}^{\alpha}$ and $\mathbf{q}^{\alpha}$ are the momentum flux and heat flux due to the homogeneous deformation of lattice cells; $\tau^{\alpha}$ and $\mathbf{j}^{\alpha}$ are the momentum flux and heat flux resulting from the reorganizations of atoms within the lattice cells.

Governing equations of CAC are numerically solved using a finite element method. CAC resolves full atomistic resolution in the vicinity of interfaces while coarse graining to thousands of atoms per elements in regions away from interfaces. Therefore, CAC is able to significantly reduce the DOFs of the computer model while maintaining the accuracy of interfacial structures. Also, with the interatomic potential being the only constitutive law, CAC does not require any other priori assumptions regarding phonon transport. In existing works, CAC has been demonstrated reliable in modeling and simulating dislocations, ${ }^{29,40,52-61}$ reproducing phonon 
dispersion relations ${ }^{62}$ and simulating transient phonon transport and interaction with defects including dislocations. ${ }^{54,63}$

\section{COMPUTER MODEL AND SIMULATION SETUP}

In this work, we choose an ideal material system composed of two types of FCC solids, labeled material A and B, with $20 \%$ difference in atomic mass and $4 \%$ mismatch in the lattice constant. Coarse-grained models of two-dimensional superlattices are constructed with a uniform mesh of $1.04 \mathrm{~nm}$. Each model has a single crystal buffer zone with a width of $0.31 \mu \mathrm{m}$ and two symmetric superlattice regions containing 30 periods of alternating layers of material A and B. The superlattice period thickness is $10.4 \mathrm{~nm}$, and the total dimension of the computer model is $1.04 \mu \mathrm{m} \times 1.04 \mu \mathrm{m}$. The LenardJones potential ${ }^{64}$ is employed to model atomic interactions of the two materials. Initially, lattice parameters of material A and B in model II are set as their equilibrium lattice constants. For model I with coherent interfaces, the lattice parameters of material $\mathrm{A}$ and $\mathrm{B}$ along a direction parallel to the interfaces are set as the equilibrium lattice constant for material $\mathrm{A}$, while along the direction normal to the interfaces are set as their own equilibrium lattice constants. Potential parameters are listed in Table 1. Presented in Fig. 1 are computer models after equilibration. For model I with coherent

Table 1. LJ potential parameters of material $A$ and B

\begin{tabular}{|c|c|c|}
\hline $\begin{array}{l}\text { Mass } \\
\text { ratio }\end{array}$ & $\begin{array}{c}\text { Lattice constant } \\
\text { ratio }\end{array}$ & $\begin{array}{c}\text { Dielectric constant } \\
\text { ratio }\end{array}$ \\
\hline$\frac{m_{\mathrm{A}}}{m_{\mathrm{B}}}=1.2$ & $\frac{\sigma_{\mathrm{A}}}{\sigma_{\mathrm{B}}}=1.04$ & $\frac{\varepsilon_{\mathrm{A}}}{\varepsilon_{\mathrm{B}}}=5.0$ \\
\hline
\end{tabular}

interfaces, we find large strain in the material layers as an accommodation of the lattice mismatch. In model II, arrays of defects ("misfit dislocations") are formed along interfaces after relaxation. The strain is observed to be concentrated around defects, with the regions away from interfaces almost free of strain.

The heat source with a broadband phonon wavelength is generated by using a coherent phonon pulse model ${ }^{63,65}$ to mimic the material response to ultra-fast laser pulse excitations. A displacementbased wave packet with Gaussian spatial distribution is applied as an initial condition within a circular heater region with, i.e.,

$$
\mathbf{u}(\mathbf{r}, t=0)=\sum_{j} A_{j} \mathbf{e}_{j} \exp \left(i \mathbf{k}_{j} \cdot \mathbf{r}\right) \cdot \exp \left[-\frac{|\mathbf{r}|^{2}}{\eta_{j}^{2}}\right]
$$

where $\mathbf{r}$ is the relative position vector from the material point to the center of the heat source; $A_{j}, \mathbf{e}_{j}$ and $\mathbf{k}_{j}$ are the amplitude, eigenvector and wave vector of phonon mode with the index of $j$, respectively; $\eta$ is the variance of the spatial Gaussian distribution. The phonon number is determined by the Bose-Einstein distribution. ${ }^{18}$ Phonons are naturally permitted to scatter or interfere each other after the initial time step. The phonon heat pulse is constructed of phonons with wavelength between twice the element size $2.08 \mathrm{~nm}$ and the total length of superlattice region $0.31 \mu \mathrm{m}$.

\section{SIMULATION RESULTS AND DISCUSSION}

In Fig. 2, we present time sequences of kinetic energy distribution in superlattices, showing the transient processes of heat pulse propagation and its interaction with coherent and semi-coherent interfaces; distinct phenomena are observed. As shown in Fig. 2b, a partial transmission and reflection of phonons at the first interface of the coherent superlattice region are observed at $t=30 \mathrm{ps}$. The

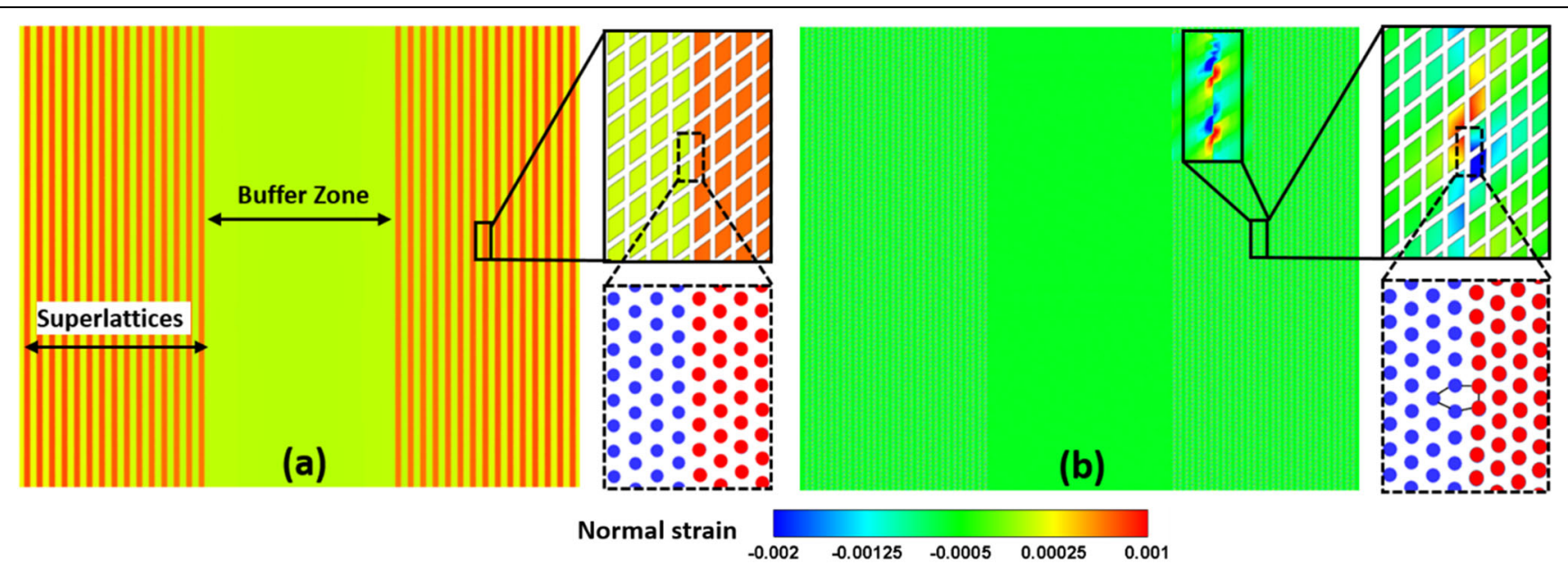

Fig. 1. Schematic presentations of two-dimensional superlattices with (a) model I: coherent interfaces; (b) model II: semi-coherent interfaces. Zoom-in figures show the finite element mesh and the resolved atomic structure in the vicinity of the interface. The color contour is the atomic strain. 
noticeable wave feature indicates that the phonon scatterings at coherent interfaces are coherent. Most of the energy "caustics" (directions with especially high phonon fluxes resulted from crystalline anisotropy) are impeded by the first interface and only a few of them transmitted across. Among the transmitted phonons, most of them propagate ballistically across the superlattices without being scattered by the interfaces as shown in Fig. 2c; this indicates that phonons have been converted into eigenmodes of vibration that are compatible with superlattices after the transmission across the first interface. At $t=70 \mathrm{ps}$, we find an appreciable portion of energy confined within the single-crystal region, while only a very small fraction of energy is "trapped" in the superlattice region. In contrast, the phonon transport in superlattices containing interface defects behaves differently, as shown in Fig. 2e, f, $g$ and $h$. Unlike the ballistic transport in the coherent superlattice region as presented in Fig. 2b, c and d, the majority of phonons are strongly scattered by semi-coherent interfaces, as shown in Fig. $2 f$ and g. At $t=70 \mathrm{ps}$, there is a significant amount of kinetic energy left in the superlattice region.

To quantify the effect of interface defects on phonon transport, we calculate the kinetic energy transmissions across the first interface (defined as region I) and the superlattice region (defined as region II) as functions of simulation time in both models. Energy transmission is calculated as

$$
\alpha_{\mathrm{D}}(t)=\frac{E_{0}-E_{\mathrm{D}}(t)}{E_{0}}
$$
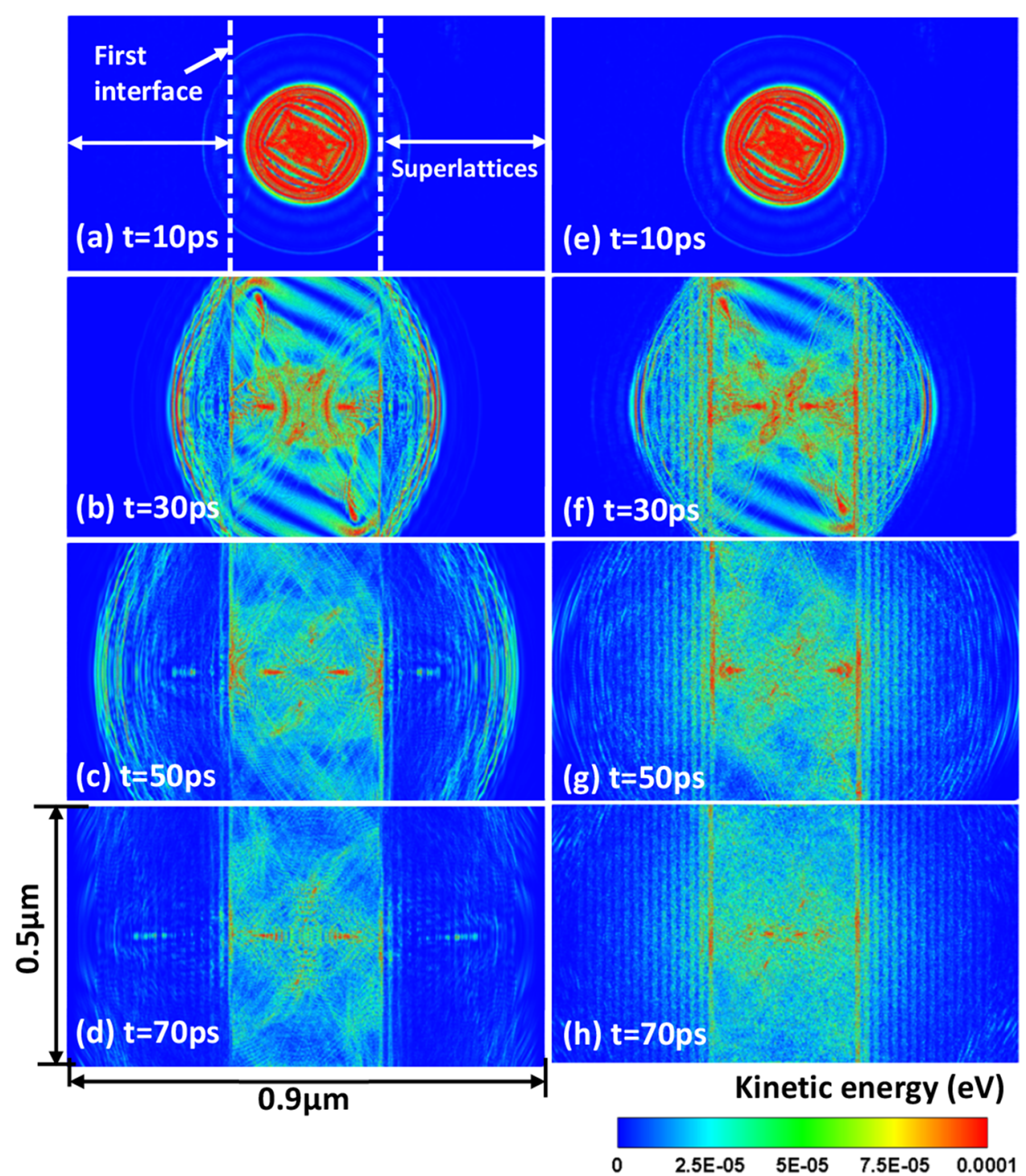

Fig. 2. Time sequences of kinetic energy distributions from $t=10 \mathrm{ps}$ to $t=70 \mathrm{ps}$ in models with (a)-(d) coherent interfaces; (e)-(h) semicoherent interfaces. The image size is $0.9 \mu \mathrm{m} \times 0.5 \mu \mathrm{m}$. 
where $E_{0}$ is the total energy input into the system by the heat pulse, $E_{\mathrm{D}}(t)$ is the energy contained within a region $\mathrm{D}$ at time $t$, and $\alpha_{\mathrm{D}}(t)$ is the energy transmission across region $\mathrm{D}$ at time $t$. Time-dependent energy transmissions for the two cases are shown in Fig. 3. First, in both coherent and incoherent superlattices, we find a rapid increase in energy transmission across region I from $t=5 \mathrm{ps}$ to $t=15 \mathrm{ps}$, during the process of which the heat pulse interacts with the first interface. Afterwards, the increase of energy transmissions slows down, and they converge. Then, a phonon wave front starts reaching the boundary of both models at $t=50 \mathrm{ps}$, and the energy transmission across region II begins to accumulate. At the end of the simulation, energy transmissions across region I and II are calculated as $47.2 \%$ and $30.0 \%$ for model I, $48.2 \%$ and $3.5 \%$ for model II. We find that the energy transmission across the first interface in model II is slightly higher than in model I, with the coherent interface providing more resistance to phonon propagation than the semi-coherent interface. It can be explained by the Bragg reflection effect, which results from the constructive interference between phonons reflected by interfaces. For model II, the phonon wave interference is destroyed by the incoherent scattering of phonons by interface defects, and thus the effect of the Bragg reflection is weakened. The energy transmission across the first interface in model $I$ is therefore lower than that in model II. Another interesting finding is that the overall energy transmission across superlattices containing interface defects is one order of magnitude lower than that in superlattices with coherent interfaces. This again indicates the difference in the phonon transport nature. In model I, $64 \%$ of phonons that transmit across the first interface can propagate across the superlattice layers without

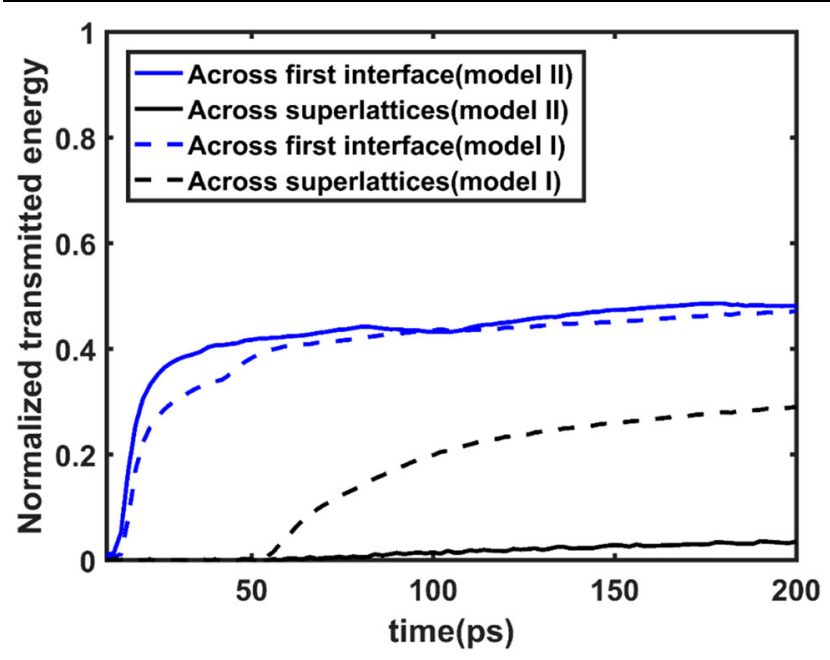

Fig. 3. Energy transmission across the first interface, denoted as region I, and superlattices, denoted as region II, in models with coherent interfaces and semi-coherent interfaces, from $t=0 \mathrm{ps}$ to $t=200 \mathrm{ps}$. being scattered. In model II, phonon defect scattering breaks down the phonon coherency and destroys phonon wave interference. Phonons are incoherently scattered by each interface and are prevented from converting into eigenmodes of superlattices. Consequently, phonon transport becomes diffusive, resulting in an ultra-low-energy transmission of $3.5 \%$.

In superlattices containing interface defects, phonons are strongly scattered by the interfaces with only a small fraction of them propagating ballistically. Note that the generated phonons have similar group velocities but different wavelengths. We then present a mode-wise study to investigate the wavelength-dependent phonon defect scattering. Phonon waves with dominant wavelengths of $43 \mathrm{~nm}, 28 \mathrm{~nm}$ and $5 \mathrm{~nm}$ are generated by applying excitation using the following equation,

$$
u(\mathbf{r}, t)=A_{j} \mathbf{e}_{j} \exp \left(i \mathbf{k}_{j} \cdot \mathbf{r}-\omega_{j} t\right)
$$

In Fig. 4, we present the comparative simulation results of propagation of monochromatic phonon waves in superlattices with and without interface defects. Phonons with 43-nm wavelength behave similarly in the two models-they transmit partially across the first interface and remain coherent in the superlattice region, with phonons in model II only slightly disturbed by interface defects. For phonons with a shorter wavelength of $28 \mathrm{~nm}$, we observe a strong phonon defect scattering in model II. When the phonon wavelength reduces to $5 \mathrm{~nm}$, we observe a qualitative difference. In model I, we find a strong anisotropy in the kinetic energy distribution in the superlattice region due to the wave interference effect. In addition, as the phonon wavelength $\lambda$ and superlattice period thickness $\mathrm{p}$ satisfy the relation $n \lambda=p$, the condition of Bragg reflection applies. This is demonstrated by simulation results, i.e., phonons propagating along the direction normal to interfaces are found to be completely reflected. In model II, phonon transport in superlattices is diffusive, and the Bragg reflection phenomenon disappears. Simulation results indicate that the phonon defect scattering becomes stronger with decreasing phonon wavelength. For phonons with a short wavelength of $\sim 5 \mathrm{~nm}$, interface defects destroy the phonon coherency and lead to a change of the phonon transport nature in superlattices from coherent to diffusive.

\section{SUMMARY}

This work demonstrates the application of the CAC method to the study of transient phonon heat transport across coherent and incoherent interfaces. Simulation results show that the defects at the incoherent interfaces affect phonon transport by two mechanisms. First, each individual defect acts as a scattering source for phonons. The high density of the interface defects leads to frequent incoherent phonon scattering events, resulting in an increase of 


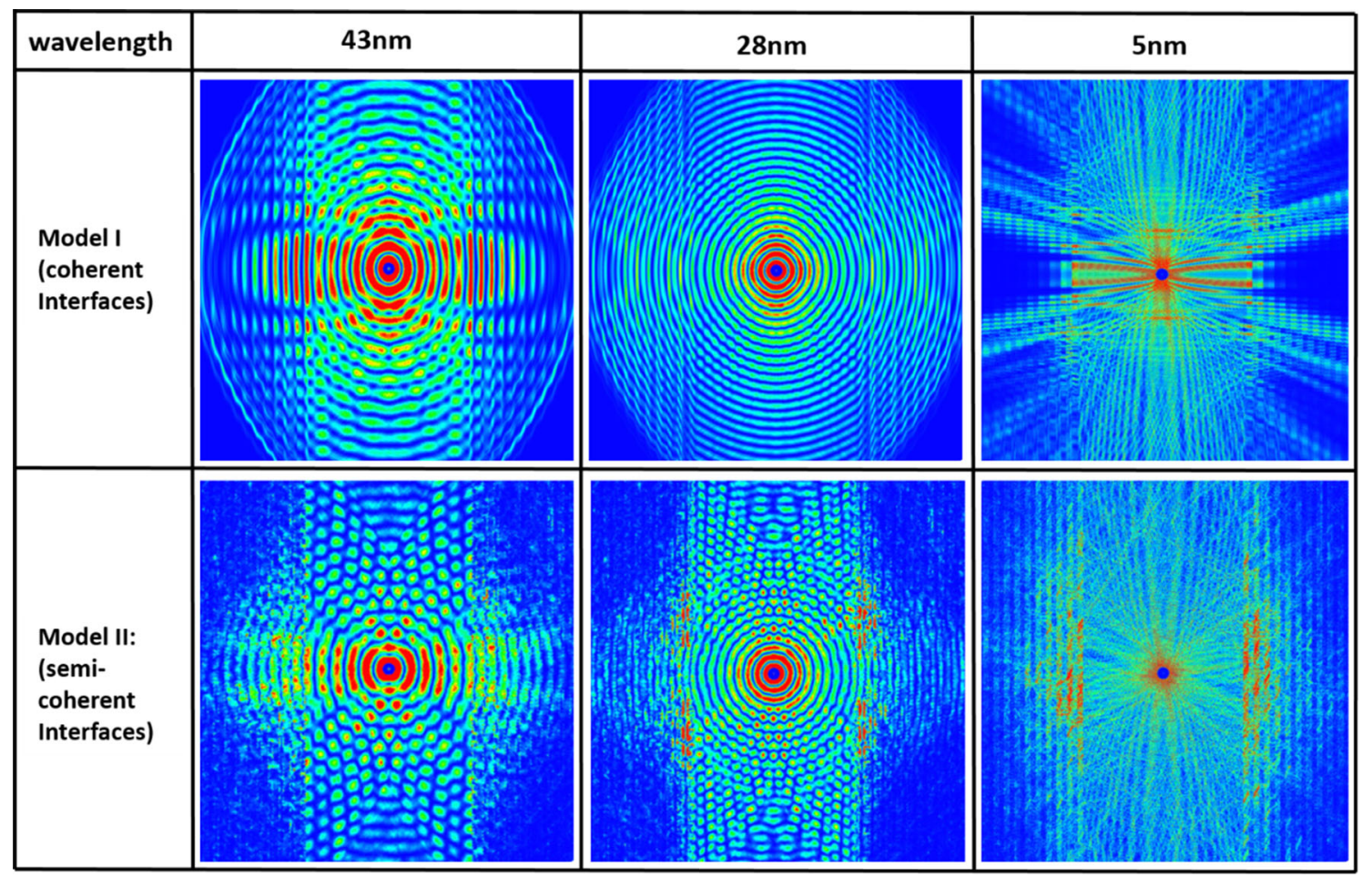

Fig. 4. Snapshots of kinetic energy distribution, representing propagations of phonon monochromatic waves with wavelengths of $5 \mathrm{~nm}$, $28 \mathrm{~nm}$ and $43 \mathrm{~nm}$ in superlattices with coherent and semi-coherent interfaces.

diffusive phonons. Second, the existence of the defects undermines the periodicity of superlattices, which results in a destruction of phonon wave coherence as well as interference. These effects then bring about a change of the phonon transport nature in superlattices from coherent to diffusive. Different from steady-state simulations, which usually interpret thermal conductivity or conductance with theoretical models, this work simulates the transient processes of phonon propagation and scattering in superlattice systems. Apart from the quantification of energy transmission, the nature of phonon transport is revealed through a direct visualization in this work. The main findings are summarized as follows:

1. Phonon transport across superlattices with coherent and semi-coherent interfaces is studied. In a superlattice with coherent interfaces, phonon transport is mainly coherent. The majority of energy transmission is from coherent phonons that propagate ballistically through the layers of superlattices.

2. The existence of defects at the semi-coherent interfaces destroys the wave interference and hence changes the phonon transport nature from coherent to diffusive. The energy transmission across superlattices containing interface defects is an order of magnitude lower than that across superlattices with coherent interfaces.
3. The mode-wise study of phonon propagation in the semi-coherent superlattices shows that the shorter wavelength phonons are more sensitive to the interface defects and the scattering between them is stronger with decreasing phonon wavelength. When the phonon wavelength reduces to $5 \mathrm{~nm}$, phonon wave interference is completely destroyed, and the phonon transport becomes diffusive.

\section{ACKNOWLEDGEMENTS}

This work is supported by the US Department of Energy, Office of Science, Basic Energy Sciences, under Award No. DESC0006539. Also, X.C. and S.Y. acknowledge Extreme Science and Engineering Discovery Environment (XSEDE) allocations TGMSS180016 and TG-MSS180015, respectively.

\section{REFERENCES}

1. P.E. Hopkins. ISRN Mech. Eng. 2013, (2013).

2. P.E. Hopkins, J.C. Duda, C.W. Petz, and J.A. Floro, Phys. Rev. B 84, 035438 (2011).

3. P.E. Hopkins, J.C. Duda, S.P. Clark, C.P. Hains, T.J. Rotter, L.M. Phinney, and G. Balakrishnan, Appl. Phys. Lett. 98, 161913 (2011).

4. J.C. Duda and P.E. Hopkins, Appl. Phys. Lett. 100, 111602 (2012).

5. B.C. Gundrum, D.G. Cahill, and R.S. Averback, Phys. Rev. B 72, 245426 (2005).

6. R.M. Costescu, M.A. Wall, and D.G. Cahill, Phys. Rev. B 67, 054302 (2003).

7. H.-K. Lyeo and D.G. Cahill, Phys. Rev. B 73, 144301 (2006). 
8. S.-M. Lee, D.G. Cahill, and R. Venkatasubramanian, Appl. Phys. Lett. 70, 2957 (1997).

9. T. Zhu and E. Ertekin, Phys. Rev. B 90, 195209 (2014).

10. A. Hanisch-Blicharski, B. Krenzer, S. Wall, A. Kalus, T. Frigge, and M.H.-V. Hoegen, J. Mater. Res. 27, 2718 (2012).

11. P.E. Hopkins, P.M. Norris, R.J. Stevens, T.E. Beechem, and S. Graham, J. Heat Transf. 130, 062402 (2008).

12. J. Ravichandran, A.K. Yadav, R. Cheaito, P.B. Rossen, A. Soukiassian, S.J. Suresha, J.C. Duda, B.M. Foley, C.-H. Lee, Y. Zhu, A.W. Lichtenberger, J.E. Moore, D.A. Muller, D.G. Schlom, P.E. Hopkins, A. Majumdar, R. Ramesh, and M.A. Zurbuchen, Nat. Mater. 13, 168 (2014).

13. D.L. Yunfei Chen, J.R. Lukes, Z. Ni, and M. Chen, Phys. Rev. B 72, 174302 (2005).

14. G.D.M.M.V. Simkin, Phys. Rev. Lett. 84, 927 (2000).

15. J.P. Wolfe, Imaging Phonons: Acoustic Wave Propagation in Solids (New York: Cambridge University Press, 2005).

16. S. Sadasivam, Y. Che, Z. Huang, L. Chen, S. Kumar, and T.S. Fisher, Annu. Rev. Heat. Trans. 17, 89 (2014).

17. A. Chernatynskiy, D. Clarke, and S. Phillpot, Thermal transport in nanostructured materials.Handbook of $\mathrm{Na}$ noscience, Engineering, and Technology, 3rd ed., ed. W.A. Goddard, D. Brenner, S.E. Lyshevski, and G.J. Iafrate (Boca Rotan: CRC Press, 2012), p. 545.

18. M.T. Dove, Introduction to Lattice Dynamics (Cambridge: Cambridge University Press, 1993).

19. X.W. Zhou, R.E. Jones, and S. Aubry, Phys. Rev. B 81, 155321 (2010).

20. K.T. Regner, D.P. Sellan, Z. Su, C.H. Amon, A.J.H. McGaughey, and J.A. Malen, Nat. Commun. 4, 1640 (2013).

21. L. Chen, J.L. Braun, B.F. Donovan, P.E. Hopkins, and S.J. Poon, Appl. Phys. Lett. 111, 131902 (2017).

22. M. Maldovan, Nat. Mater. 14, 667 (2015).

23. Y. Chen and J. Lee, Philos. Mag. 85, 4095 (2005).

24. Y. Chen, J. Chem. Phys. 124, 054113 (2006).

25. Y. Chen, J. Chem. Phys. 130, 134706 (2009).

26. Y. Chen, J. Zimmerman, A. Krivtsov, and D.L. McDowell, Int. J. Eng. Sci. 49, 1337 (2011).

27. L. Xiong, A Concurrent Atomistic-Continuum Methodology and its Applications (Gainesville: University of Florida, 2011).

28. Q. Deng, Coarse-Graining Atomistic Dynamics of Fracture by Finite Element Method Formulation, Parallelization and Applications (Gainesville: University of Florida, 2011).

29. S. Yang, A Concurrent Atomistic-Continuum Method for Simulating Defects in Ionic Materials (Gainesville: University of Florida, 2014).

30. S. Xu, T.G. Payne, H. Chen, Y. Liu, L. Xiong, Y. Chen, and D.L. McDowell, J. Mater. Res. 33, 857 (2018).

31. S. Xu, R. Che, L. Xiong, Y. Chen, and D.L. McDowell, Int. J. Plast. 72, 91 (2015).

32. A. Diaz, D. McDowell, and Y. Chen, The limitations and successes of concurrent dynamic multiscale modeling methods at the mesoscale.Generalized Models and Nonclassical Approaches in Complex Materials, Vol. 2, ed. H. Altenbach, J. Pouget, M. Rousseau, B. Collet, and T. Michelitsch (Berlin: Springer, 2018), p. 55.

33. S. Xu, L. Xiong, Y. Chen, and D.L. McDowell. JOM, 1 (2017).

34. S. Xu, L. Xiong, Y. Chen, and D. McDowell, Crystals 7, 120 (2017).

35. X. Chen, L. Xiong, D.L. McDowell, and Y. Chen, Scr. Mater. 137, 22 (2017).

36. X. Chen, W. Li, L. Xiong, Y. Li, S. Yang, Z. Zheng, D.L. McDowell, and Y. Chen, Acta Mater. 136, 355 (2017).
37. P.A. Pluchino, X. Chen, M. Garcia, L. Xiong, D.L. McDowell, and Y. Chen, Comput. Mater. Sci. 111, 1 (2016).

38. S. Xu, L. Xiong, Y. Chen, and D.L. McDowell, NPJ Comput. Mater. 2, 15016 (2016).

39. L. Xiong, J. Rigelesaiyin, X. Chen, S. Xu, D.L. McDowell, and Y. Chen, Acta Mater. 104, 143 (2016).

40. S. Xu, L. Xiong, Y. Chen, and D.L. McDowell, Scr. Mater. 123, 135 (2016).

41. S. Xu, L. Xiong, Y. Chen, and D.L. McDowell, J. Mech. Phys. Solids 96, 460 (2016).

42. L. Xiong, S. Xu, D.L. McDowell, and Y. Chen, Int. J. Plast. 65, 33 (2015)

43. L. Xiong, D.L. McDowell, and Y. Chen, Int. J. Plast. 55, 268 (2014).

44. L. Xiong, Q. Deng, G. Tucker, D.L. McDowell, and Y. Chen, Acta Mater. 60, 899 (2012).

45. L. Xiong, Q. Deng, G.J. Tucker, D.L. McDowell, and Y. Chen, Int. J. Plast. 38, 86 (2012).

46. L. Xiong, D.L. McDowell, and Y. Chen, Scr. Mater. 67, 633 (2012).

47. L. Xiong, G. Tucker, D.L. McDowell, and Y. Chen, J. Mech. Phys. Solids 59, 160 (2011).

48. J.H. Irving and J.G. Kirkwood, J. Chem. Phys. 18, 817 (1950).

49. J.G. Kirkwood, J. Chem. Phys. 14, 180 (1946).

50. C. Kittel, Introduction to Solid State Physics (Hoboken: Wiley, 1956).

51. Y. Chen, J. Chem. Phys. 130, 134706 (2009).

52. L. Xiong, S. Xu, D.L. McDowell, and Y. Chen, Int. J. Plast. 65,33 (2015).

53. S. Xu, L. Xiong, Y. Chen, and D.L. McDowell, npj Comput. Mater. 2, 15016 (2016).

54. X. Chen, L. Xiong, D.L. McDowell, and Y. Chen, Scri. Mater. 137, 22 (2017).

55. L. Xiong, Q. Deng, and Y. Chen, Coarse-grained atomistic simulations of dislocation and fracture in metallic materials.Handbook of Micromechanics and Nanomechanics, ed. S. Li and X.L. Gao (Stanford: Pan Stanford Publishing, 2013),

56. L. Xiong, Q. Deng, G. Tucker, D.L. McDowell, and Y. Chen, Acta Mater. 60, 899 (2012).

57. L. Xiong, Q. Deng, G.J. Tucker, D.L. McDowell, and Y. Chen, Int. J. Plast. 38, 86 (2012).

58. S. Yang, L. Xiong, Q. Deng, and Y. Chen, Acta Mater. 61, 89 (2013).

59. S. Yang and Y. Chen, Proc. R. Soc. A 471, 20140758 (2015)

60. S. Yang, N. Zhang, and Y. Chen, Philos. Mag. 95, 2697 (2015).

61. S. Yang and Y. Chen, Concurrent atomistic-continuum simulation of defects in polyatomic ionic materials.Multiscale Materials Modeling for Nanomechanics, ed. C.R. Weinberger and G.J. Tucker (Berlin: Springer, 2016), p. 261.

62. L. Xiong, X. Chen, N. Zhang, D.L. McDowell, and Y. Chen, Arch. Appl. Mech. 84, 1665 (2014).

63. X. Chen, W. Li, L. Xiong, Y. Li, S. Yang, Z. Zheng, D.L. McDowell and Y. Chen. Acta Mater. 136, 355 (2017).

64. Y. Kogure, T. Tsuchiya, and Y. Hiki, J. Phys. Soc. Jpn. 56, 989 (1987).

65. X. Chen, A. Chernatynskiy, L. Xiong, and Y. Chen, Comput. Phys. Commun. 195, 112 (2015).

Publisher's Note Springer Nature remains neutral with regard to jurisdictional claims in published maps and institutional affiliations. 